\title{
Mg-Sm (Magnesium-Samarium)
}

\author{
H. Okamoto
}

The $\mathrm{Mg}-\mathrm{Sm}$ phase diagram in [Massalski2] was redrawn from [1989Sac]. The phase relationships among Sm allotropes were uncertain. Figure 1 shows the $\mathrm{Mg-Sm}$ phase diagram calculated by [2008Jia] based primarily on the work of [1989 Sac]. Possible relationships among Sm allotropes are shown.

$\mathrm{Mg}-\mathrm{Sm}$ crystal structure data given in Table 1 were adopted from [Massalski2] with the composition ranges modified according to [2008Jia].

\section{References}

1989Sac: A. Saccone, S. Delfino, G. Borzone, and R. Ferro, The Samarium-Magnesium System: A Phase Diagram, J. LessCommon Met., 1989, 154, p 47-60

2008Jia: B.R. Jia, L.B. Liu, D.Q. Yi, Z.P. Jin, and J.F. Nie, Thermodynamic Assessment of the Al-Mg-Sm System, J. Alloys Compds., 2008, 459, p 267-273

Table 1 Mg-Sm crystal structure data

\begin{tabular}{|c|c|c|c|c|c|}
\hline Phase & Composition, at.\% Sm & Pearson symbol & Space group & Strukturbericht designation & Prototype \\
\hline$(\mathrm{Mg})$ & 0 & $h P 2$ & $P 6_{3} / m m c$ & $A 3$ & $\mathrm{Mg}$ \\
\hline $\mathrm{Mg}_{41} \mathrm{Sm}_{5}$ & 10.9 & $t 192$ & $I 4 / m$ & $\ldots$ & $\ldots$ \\
\hline $\mathrm{Mg}_{5} \mathrm{Sm}$ & 16.7 & $c F 444$ & $F \overline{4} 3 m$ & $\ldots$ & $\ldots$ \\
\hline $\mathrm{Mg}_{3} \mathrm{Sm}$ & 25 & $c F 16$ & $F m \overline{3} m$ & $\mathrm{DO}_{3}$ & $\mathrm{BiF}_{3}$ \\
\hline $\mathrm{Mg}_{2} \mathrm{Sm}$ & 33.3 & $c F 24$ & $F d \overline{3} m$ & $C 15$ & $\mathrm{Cu}_{2} \mathrm{Mg}$ \\
\hline $\mathrm{MgSm}$ & 50 & $c P 2$ & $P m \overline{3} m$ & $B 2$ & $\mathrm{CsCl}$ \\
\hline$(\gamma \mathrm{Sm})$ & $69.7-100$ & $c / 2$ & $\operatorname{Im} \overline{3} m$ & $A 2$ & $\mathrm{~W}$ \\
\hline$(\beta \mathrm{Sm})$ & $94.2-100$ & $h P 2$ & $P 6_{3} / m m c$ & $A 3$ & $\mathrm{Mg}$ \\
\hline$(\alpha \mathrm{Sm})$ & $97.15-100$ & $h R 3$ & $R \overline{3} m$ & $\ldots$ & $\alpha \mathrm{Sm}$ \\
\hline
\end{tabular}

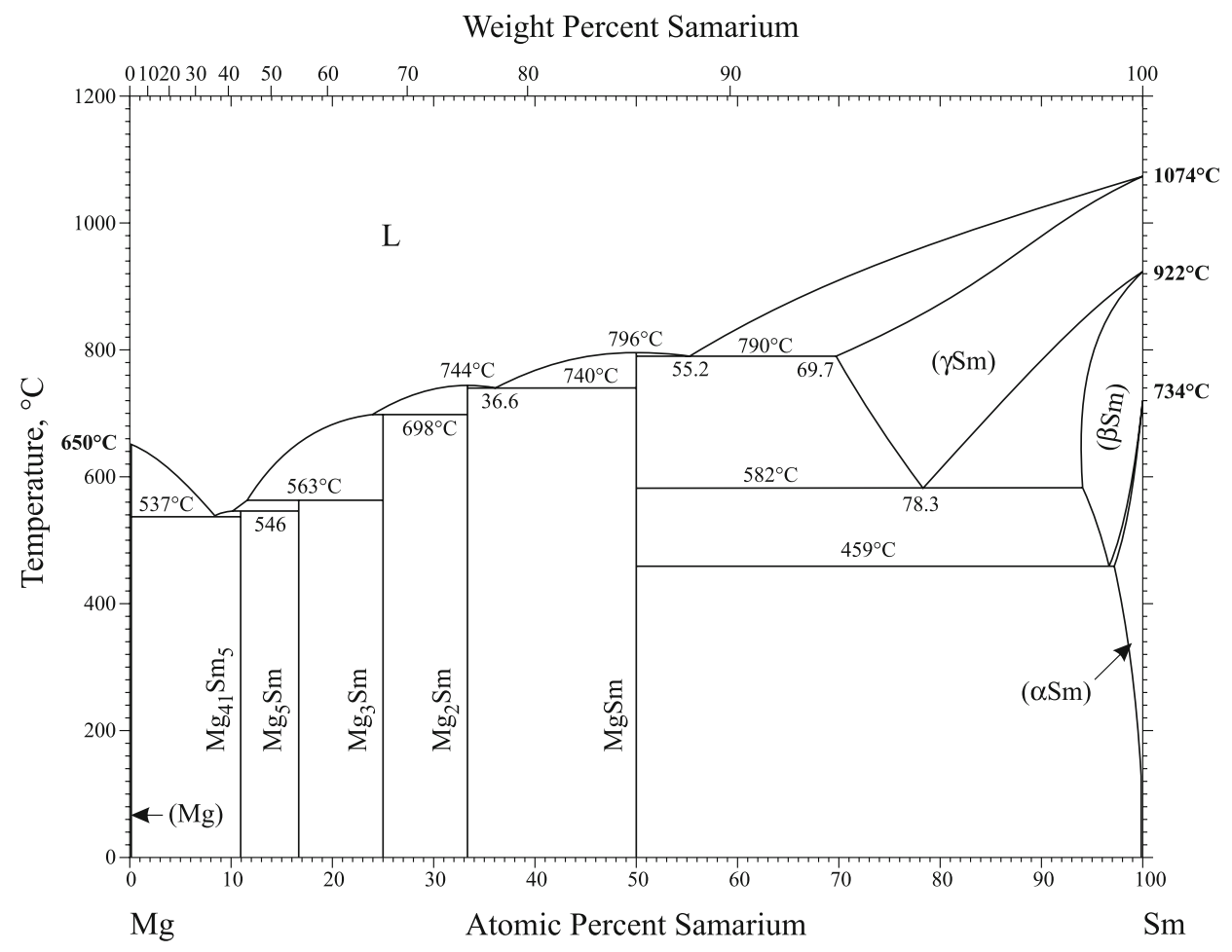

Fig. $1 \mathrm{Mg}$-Sm phase diagram 